\title{
Design and Development of Collaboration Technologies
}

\author{
Douglas C. Derrick \\ University of Nebraska at \\ Omaha \\ dcderrick@unomaha.edu
}

\author{
Ryan M. Schuetzler \\ University of Nebraska at \\ Omaha \\ rschuetzler@unomaha.edu
}

\author{
G. Mark Grimes \\ University of Houston \\ gmgrimes@bauer.uh.edu
}

Collaboration has long been one of the primary uses of information systems. In fact, among the first applications of network technologies was to transmit text-based messages between users. As technology has evolved, so have computer-mediated communication and collaboration. From new methods for interacting with computers, such as voice interactions, to new methods for users to collaborate, such as through social media, the landscape in which people collaborate is rapidly evolving.

Matching collaboration technologies to tasks and users is critical for improving performance and satisfaction. Thus, designing and developing collaboration technology requires consideration of the individuals, the communication processes, the nature of the problem, and the domain/context. Proper fit of collaboration technologies to the task and context leads to increased communication performance which then leads to increased task performance. Proper fit is dependent not only on an individual's experience but the goal of the collaborative communication (e.g., achieving convergence among those communicating or conveying information). In other words, the appropriateness of collaboration technologies for a group or task is mediated by the information processing needs and goals of the users.

Given the importance of collaboration in day-to-day life, this mini-track explores vital issues around technologies used for collaboration. The mini-track provides a platform to explore how technological innovation and new processes are reshaping computermediated human-to-human collaboration. Specifically, this mini-track addresses the following topics:

1. Theoretical foundations and design methodologies for collaborative work practices and technologies

2. Processes and tools for establishing and maintaining shared focus and shared mental models over time

3. Processes, technologies, and theoretical breakthroughs to improve and speed up shared sense-making
4. Methods and technologies for eliciting and capturing tacit knowledge from experts (i.e., externalization) and sharing / incorporating that knowledge into collaborative efforts (i.e., team internalization)

5. Facilitation methods, techniques, patterns, and procedures to improve (a)synchronous collaboration between co-located and distributed people, teams, or groups

6. Assessment models and methods for team collaboration and performance

7. Design, codification, and reuse of work practices and pattern languages for group collaboration

8. Design and building of automated virtual agents to participate in online collaborations (e.g., ChatOps)

This year we have excellent papers covering the design and creation of collaboration technology. The first, "Towards Trust-Aware Human-Automation Interaction: An Overview of the Potential of Computational Trust Models," investigates computational models for trust in the design and deployment phases of the creation of automated technology. The second paper, "From Voice to Knowledge: A Proposal for a Voice Annotation System to Support Collaborative Engineering Design Processes," describes the creation of a voice interaction tool to assist with collaborative Computer-Aided Design (CAD) processes. The final paper in the mini track is "Capturing the Forest or the Trees: Designing for Granularity in Data Crowdsourcing," which examines considerations for the design of data crowdsourcing projects, focusing on the relative depth of information crowds can provide.

We thank the authors for their effort in preparing these excellent manuscripts for the conference. We hope you enjoy the papers and presentations at the conference and look forward to more insights for enabling collaborative work. 